\title{
Characterization of SnTe Films Grown by Molecular Beam Epitaxy
}

\author{
U. A. Mengui, E. Abramof, P. H. O. Rappl, and A Y. Ueta \\ Instituto Nacional de Pesquisas Espaciais - INPE, Laboratório Associado de Sensores e Materiais - LAS, \\ Caixa Postal 515, 12245-970, São José dos Campos - SP, Brazil.
}

Received on 4 April, 2005

\begin{abstract}
A series of SnTe layers with thicknesses varying from 0.42 to $9.1 \mu \mathrm{m}$ were grown by molecular beam epitaxy on (111) $\mathrm{BaF}_{2}$ substrates. The SnTe lattice parameter was found to be $6.331 \AA$ as determined from x-ray diffraction spectra measured in the triple-axis configuration. The FWHM of the (222) SnTe x-ray rocking curves indicated a good crystalline quality and an unusual dependence on layer thickness. Atomic force microscopy (AFM) of the SnTe surface revealed spirals with monolayer steps formed around threading dislocations, similar to the $\mathrm{PbTe}$ on $\mathrm{BaF}_{2}$ epitaxy. The dislocation density was estimated from the AFM picture to be $9 \times 10^{8}$ $\mathrm{cm}^{-2}$. Small black pits corresponding to holes that were left during growth were also observed on the AFM images. Sn diffusion can be a possible reason for these pits and the relatively high dislocation density. Electrical measurements showed that the SnTe epilayers present a typical p-type carrier concentration around $10^{20} \mathrm{~cm}^{-3}$ almost temperature independent and a Hall mobility which decreases from $10^{4}$ to $10^{3} \mathrm{~cm}^{2} / \mathrm{V}$.s as the temperature increases from 10 to $350 \mathrm{~K}$.
\end{abstract}

Keywords: SnTe; Molecular beam epitaxy; $\mathrm{BaF}_{2}$ substrates

\section{INTRODUCTION}

SnTe is an IV-VI narrow gap semiconductor that crystallizes in the rock salt structure and whose optical and electrical properties have been investigated since the beginning of 1960's [1-3]. This kind of compound finds application in the construction of thermoelectric generators and infrared detectors [4-5]. The study of structural properties of IV-VI compounds related to misfit and threading dislocations in systems like $\mathrm{PbTe}, \mathrm{PbSe}$ and $\mathrm{PbTe}_{1-x} \mathrm{Se}_{x}$ deposited on different substrates, such as $\mathrm{KCl}, \mathrm{BaF}_{2}, \mathrm{Si}$ and $\mathrm{PbSe}$ have been done by several groups [6-10].

We investigate here the structural and electrical properties of a series of SnTe films with different thicknesses grown by molecular beam epitaxy on (111) $\mathrm{BaF}_{2}$ substrates. Highresolution x-ray diffraction and atomic force microscopy applied to the grown samples allowed the determination of structural parameters like lattice constant, crystalline quality, threading dislocation density and surface morphology. Hall concentration and mobility were determined as a function of temperature for the different SnTe films.

\section{EXPERIMENTAL PROCEDURES}

For this investigation, a series of SnTe films was grown on freshly cleaved (111) $\mathrm{BaF}_{2}$ substrates by molecular beam epitaxy in a Riber 32P MBE system. Prior to growth, the substrates were preheated at $200{ }^{\circ} \mathrm{C}$ during $30 \mathrm{~min}$, in the preparation chamber, and at $500{ }^{\circ} \mathrm{C}$ during $15 \mathrm{~min}$, in the main chamber. The layers were grown from a SnTe stoichiometric solid source. The substrate and SnTe effusion cell temperatures were kept constant during growth at $320^{\circ} \mathrm{C}$ and $676^{\circ} \mathrm{C}$, respectively, and the deposition time was varied from 0.5 to 9 h. The mean value of growth rate was found to be $0.9 \mu \mathrm{m} / \mathrm{h}$. The substrate holder was kept rotating to insure a reasonable thickness homogeneity for all samples.

A $12 \mathrm{keV}$ reflection high energy electron diffraction
(RHEED) system was used to monitor in situ the growths. After the opening of the SnTe cell shutter, the RHEED pattern immediately changes to the one characteristic of three-dimensional island formation, indicating Volmer-Weber growth mode. Subsequently, as the islands start to coalesce, the RHEED diffraction pattern changes to a streaked one and persists until the end of growth.

The thickness of the SnTe layers were determined with an Alpha Step 500 DekTak profilometer after an etch step to remove a small part of the film. The SnTe samples were structurally characterized by high-resolution x-ray diffraction measurements carried out in a Philips X'Pert diffractometer using $\mathrm{CuK} \alpha_{1}$ radiation. A four-crystal $\mathrm{Ge}(220)$ monochromator is positioned in front of the $\mathrm{x}$-ray tube, leading to an incident beam with an axial divergence of 12 arcsec and a wavelength dispersion of approximately $2 \times 10^{-4}$. The diffracted beam can go directly to the detector with an aperture of $1^{\circ}$ (open-detector configuration) or pass through a channel-cut analyzer placed in front of the detector, which reduces the detector acceptance to 12 arcsec (triple-axis configuration). An atomic force microscope (AFM) SPM2-Shimadzu operated in contact mode was used for topographical images. Finally, an automated Keithley 180A Hall effect system was used to measure the electrical properties of the samples contacted in the Van der Pauw geometry. Resistivity and Hall measurements were performed at temperature varying from 10 to $350 \mathrm{~K}$.

\section{RESULTS AND DISCUSSIONS}

We first measured the $\omega / 2 \Theta$ scans around the (222) Bragg diffraction peak of both film and substrate of all samples with the x-ray diffractometer in the triple-axis configuration. Fig. 1 shows, as an example, the x-ray diffraction spectrum of the SnTe sample with a film thickness of $1.6 \mu \mathrm{m}$. The (222) Bragg peak of the SnTe film and $\mathrm{BaF}_{2}$ substrate are clearly seen in the graph. From the angular separation between both peaks, the SnTe lattice constant was determined using the $\mathrm{BaF}_{2}$ sub- 
strate peak as a reference. The SnTe lattice parameter of almost all samples is displayed in Table I, presenting a mean value of $6.331 \AA$.

TABLE I: Data of the SnTe samples as obtained from growth parameters, profilometry and $\mathrm{x}$-ray analysis.

\begin{tabular}{|c|c|c|c|}
\hline $\begin{array}{c}\text { Thickness } \\
(\mu \mathrm{m})\end{array}$ & $\begin{array}{c}\text { Deposition } \\
\text { time }(\mathrm{h})\end{array}$ & $\begin{array}{c}\text { Lattice } \\
\text { parameter }(\AA)\end{array}$ & $\begin{array}{c}\text { FWHM } \\
(\operatorname{arcsec})\end{array}$ \\
\hline 0.42 & 0.5 & 6.332 & 201 \\
\hline 0.62 & 0.75 & 6.329 & 173 \\
\hline 0.77 & 1 & 6.333 & 254 \\
\hline 1.27 & 1 & 6.331 & 251 \\
\hline 1.60 & 2 & 6.331 & 161 \\
\hline 3.10 & 4 & 6.331 & 182 \\
\hline 4.40 & 6 & 6.332 & 179 \\
\hline 7.09 & 7.5 & - & 214 \\
\hline 9.07 & 9 & - & 231 \\
\hline
\end{tabular}

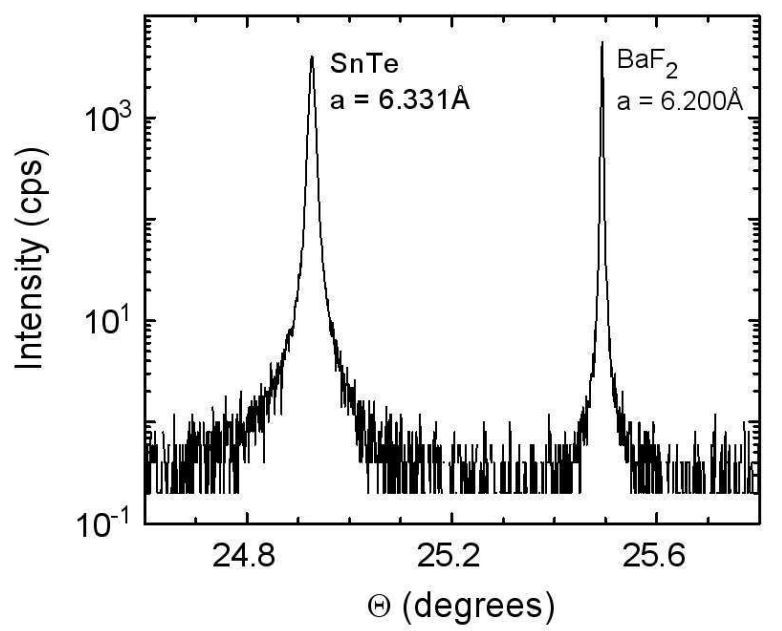

FIG. 1: $\omega / 2 \Theta$ scan measured around the (222) Bragg diffraction peak for a $\mathrm{SnTe} / \mathrm{BaF}_{2}$ sample with thickness of $1.6 \mu \mathrm{m}$.

In order to investigate the crystalline quality of the grown layers, we measured the rocking curves in the vicinity of the SnTe (222) Bragg reflex. The rocking curve is a $\omega$-scan with the open detector fixed at the desired $2 \Theta$ Bragg angle. The full-width at half-maximum (FWHM) of the rocking curve is very sensitive to threading dislocations and defects present in the film, and can be considered as a measure of the crystalline quality. The FWHM obtained for all samples is also shown in Table I, and Fig. 2 plots its dependence on the layer thickness. The FWHM varies in a range from 160 to 250 arcsec for layer thickness between 0.4 and $2.0 \mu \mathrm{m}$, and increases monotonically from 160 to 230 arcsec as the layer increases its thickness until $9 \mu \mathrm{m}$. This behavior is opposed to what is expected for this type of epitaxial layer, as in the case of $\mathrm{PbTe}_{\mathrm{T}}$ on $\mathrm{BaF}_{2}$, in which the density of threading dislocations, and hence the FWHM, decreases as the film becomes thicker [7]. We think that $\mathrm{Sn}$ diffusion may be one reason to explain this unusual behavior. Since Sn diffusion coefficient is higher in comparison, for instance, with the $\mathrm{Pb}$ one, the diffusion of $\mathrm{Sn}$ atoms during growth may lead to other type of defects that may be responsible for the rise in the FWHM values. This is especially important at relatively high growth temperatures.

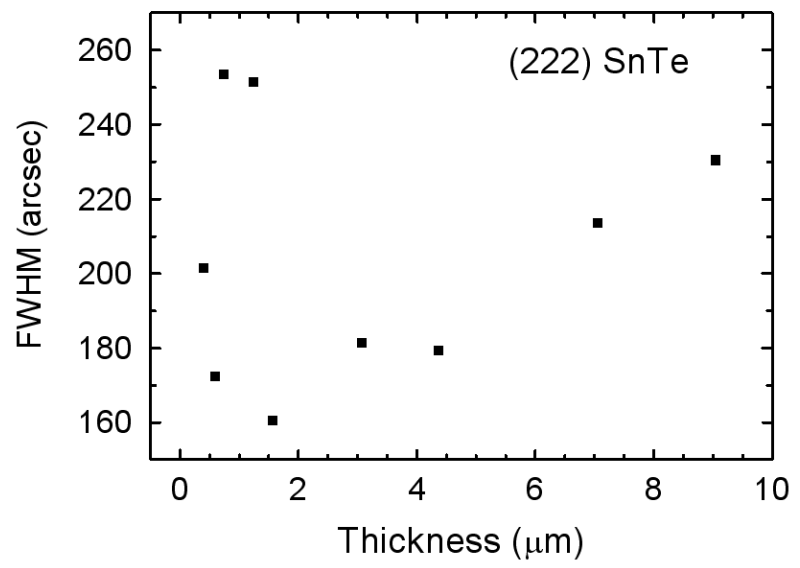

FIG. 2: Full-width at half-maximum of the (222) SnTe x-ray rocking curves as a function of layer thickness.

In order to get a better insight in this problem, we investigated the surface of the SnTe layers with atomic force microscopy. Fig. 3 shows the AFM image of a SnTe layer with a thickness of $7.09 \mu \mathrm{m}$. The scanned area is $5 \times 5 \mu \mathrm{m}^{2}$. One can clearly observe the spirals with monolayer steps near the threading dislocations, similar to the $\mathrm{PbTe}$ on $\mathrm{BaF}_{2}$ growth. Spiral growth AFM images have been reported for $\mathrm{PbTe}$ grown by $\mathrm{MBE}$ on $\mathrm{BaF}_{2}$ substrate [7,11]. It was shown that $\mathrm{PbTe}$ growth is totally dominated by spirals formed around threading dislocations that originate from the $4.2 \%$ lattice mismatch to the substrate. It is important to remind that due to the heteroepitaxy of a thin film on lattice-mismatched substrate, growth spirals normally appear as a consequence of the high density of threading dislocations formed during the strain relaxation process [12-13]. As shown in the picture of Fig. 3, the SnTe film surface actually presents a mound formation with spiral shape. The SnTe spiral heights are some few monolayer steps, and the surface of this film as a whole can be considered very smooth. We can also conclude that a pure step flow growth mode occurs in the $\mathrm{SnTe} / \mathrm{BaF}_{2}$ system, where the deposition of adatoms results in a lateral growth at the step edges.

Both ends of the short segments of monolayer steps are terminated by a threading dislocation, originated from the $2 \%$ lattice mismatch between $\mathrm{SnTe}$ and $\mathrm{BaF}_{2}$. Considering this fact, the density of dislocations can be estimated from the AFM image in Fig. 3. A value of $9 \times 10^{8} \mathrm{~cm}^{-2}$ is found for the $7.09 \mu \mathrm{m}$ thick SnTe film, which is much higher than $5 \times 10^{6}$ $\mathrm{cm}^{-2}$ reported for $\mathrm{PbTe} / \mathrm{BaF}_{2}$ film with equivalent thickness [7]. Since the lattice mismatch is smaller in the SnTe case, a smaller value for the dislocation density would be expected. Some small black pits can also be observed on the AFM image of the SnTe surface. These pits are holes that were left 


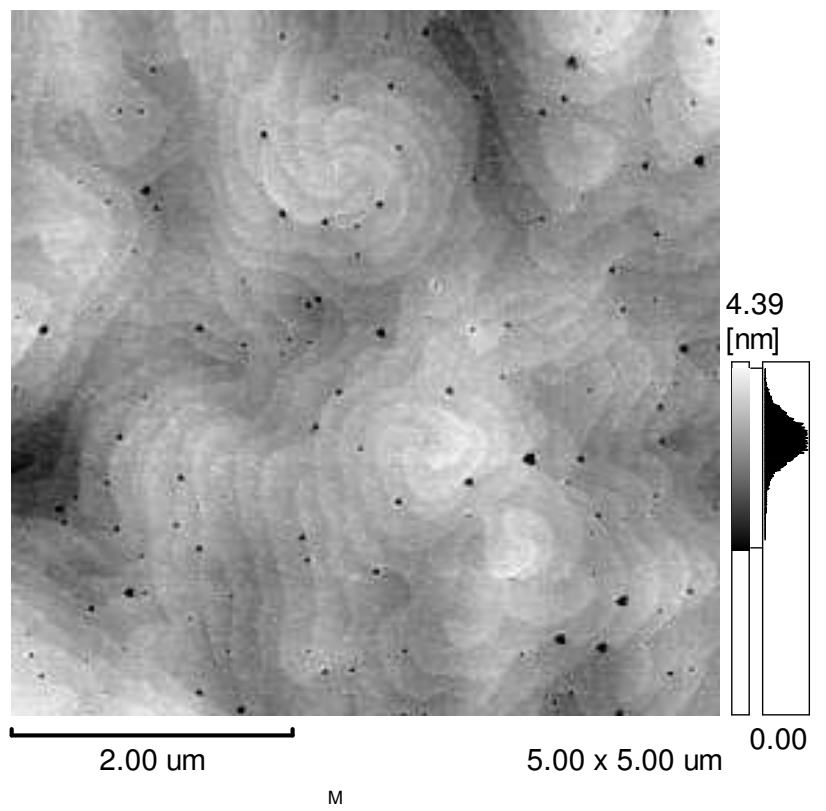

FIG. 3: AFM image of a $7.09 \mu \mathrm{m}$ thick SnTe layer grown on (111) $\mathrm{BaF}_{2}$ substrate.

during growth. We believe that Sn diffusion may be the reason for these pits and also for the higher density of threading dislocations, which lead to the relatively high FWHM values observed here in the SnTe x-ray rocking curves.

The electrical properties of the SnTe films were also measured as function of temperature from 10 to $350 \mathrm{~K}$. As for the bulk crystals, the SnTe films exhibited a high hole concentrations around $10^{20} \mathrm{~cm}^{-3}$, as shown in Fig. 4a. The strong p-type character comes from the SnTe phase diagram, which solubility is only restricted to the Te-rich region. The carrier concentration has almost no temperature dependence, as predicted from the vacancy model, showing no carrier frozen. These $p$ values are in agreement with those reported in the literature [14-16], where the lowest hole concentration obtained for SnTe films grown by MBE was $10^{19} \mathrm{~cm}^{-3}$ using special growth conditions [16] or Bi doping, which is an ntype dopant [14]. Fig. 4b shows the mobility of the SnTe films with different thickness as a function of temperature. For most of the films, the mobility decreases from $10^{4}$ to $10^{3} \mathrm{~cm}^{2} / \mathrm{V}$.s as the temperature increases from 10 to $350 \mathrm{~K}$. Phonon scattering is the main mechanism responsible for this behavior. The graph in Fig. 4b also shows that thicker SnTe films exhibited higher mobility, as expected.

\section{CONCLUSION}

A lattice parameter of $6.331 \AA$ was found for the SnTe layers grown by $\mathrm{MBE}$ on $\mathrm{BaF}_{2}$ substrates. The FWHM of the
(222) SnTe x-ray rocking curves exhibited an unusual dependence on layer thickness. The AFM image of the SnTe surface revealed spirals with monolayer steps formed around threading dislocations, similar to the $\mathrm{PbTe}$ on $\mathrm{BaF}_{2}$ epitaxy. Black
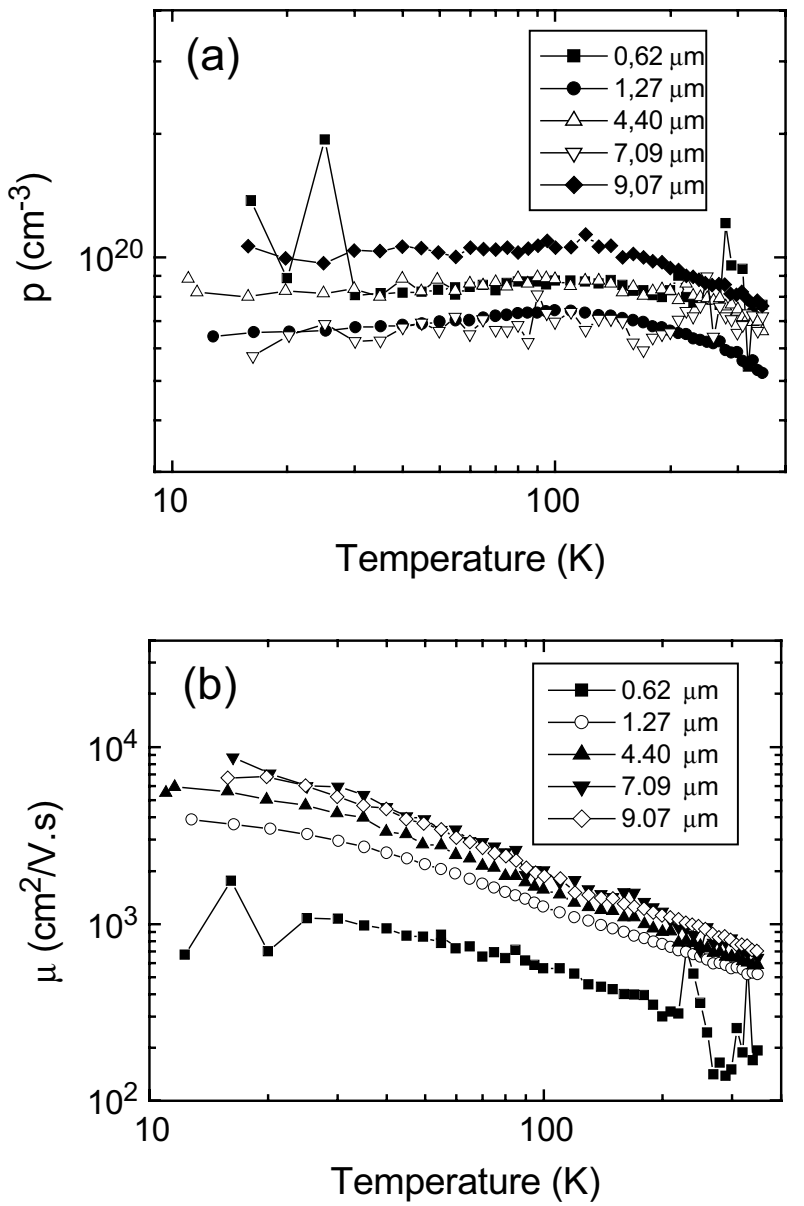

FIG. 4: Hole concentration (a) and Hall mobility (b) versus temperature for SnTe films with different thicknesses.

pits corresponding to holes created during growth were also observed on the AFM pictures. We believe that Sn diffusion may be a reason for these pits and also for the relatively high dislocation density found in the SnTe films. The carrier concentration and the Hall mobility measured for the SnTe epitaxial layers presented the expected temperature dependence and values.

\section{Acknowledgement}

The authors thank ITA/CTA in São José dos Campos, SP, Brazil, for the use of their AFM equipment. U. A. Mengui would like to thank CAPES and CNPq for financial support
[1] M. Cardona and D. L. Greenaway, Phys. Rev. 133 (6A), A1685 (1964).
[2] J. R. Burke, Jr., R. S. Allgaier, and B. B. Houston, Jr., Phys. 
Rev. Lett. 14 (10), 360 (1965).

[3] J. N. Zemel, J. D. Jensen, and R. B. Schoolar, Phys. Rev. 140 (1A), A330 (1965).

[4] G. J. Snyder, Appl. Phys. Letters 84 (13), 2436 (2004).

[5] G. Scott, C.R. Helms, J. Vacuum Science \& Technology B 9 (3): 1785-1788 (1991)

[6] A. I. Fedorenko, O. N. Nashchekina, B. A. Savitskii, L. P. Shpakovskaya, O. A. Mironov, and M. Oszwaldowski, Vacuum 43 (12), 1191 (1992).

[7] G. Springholz, A. Y. Ueta, N. Frank, and G. Bauer, Appl. Phys. Lett. 69 (19), 2822 (1996).

[8] P. Müller, H. Zogg, A. Fach, J. John, C. Paglino, A. N. Tiwari, M. Krejci, and G. Kostorz, Phys. Rev. Lett. 78 (15), 3007 (1997).

[9] H. Z. Wu, S. M. Fang, R. Salas Jr., D. McAlister, and P. J.
McCann, J. Vac. Sci. Technol. B 17 (3), 1263 (1999).

[10] K. Wiesauer and G. Springholz, Phys. Rev. B 69, 245313 (2004).

[11] A. Y. Ueta, G. Springholz, and G. Bauer, J. Cyst. Growth 175, 1022 (1997).

[12] J. Y. Tsao, Materials Fundamentals of Molecular Beam Epitaxy (Academic, London, 1993).

[13] J. W. Matthews, in Dislocation in Solids, edited by F. R. N. Nabarro (North-Holland Publ. Co, 1979).

[14] M. A. Tamor, H. Holloway, R. M. Ager, C. A. Gierczak, and R. O. Carter, J. Appl. Phys. 61 (3), 1094 (1987).

[15] A. B. Mandale, Materials Lett. 9, 389 (1990).

[16] E. Abramof, S. O. Ferreira, P. H. O. Rappl, H. Closs, and I. N. Bandeira, J. Appl. Phys. 82 (5), 2405 (1997). 